\title{
Fixateur externe zur temporären Überbrückung von Defektzonen im Unterkiefer
}

\author{
Florian Andreas Probst, Wenko Smolka, Frank Tolksdorf, Gerson Mast, \\ Carl-Peter Cornelius
}

\section{Zusammenfassung}

Die Verwendung des Fixateur externe stellt grundsätzlich eine temporäre Alternative zur internen lasttragenden Plattenosteosynthese bei Defektzonen im Unterkiefer dar. Er setzt sich aus 3 Hauptkomponenten zusammen, die allesamt aus Titan bestehen: 1) Pins oder Schanz-Schrauben, 2) vorgeformten Verbindungsstäben, 3) verstellbaren, kombinierten Klemmbacken mit Schnappmechanismus. Das Gesamtkonstrukt kann sowohl in „Visorstyle“-Technik als auch in Modulartechnik zusammengesetzt werden. Der Gebrauch ist speziellen Indikationen vorbehalten: a) pathologische Frakturen, meist im Rahmen von infizierten Osteoradionekrosen, b) weitspännige Defekte nach ablativer Tumorchirurgie, c) Notfallmanagement beispielsweise bei Polytraumapatienten mit Unterkiefertrümmerfrakturen. Unserer Erfahrung nach ist die Technik komplikationsarm und verlässlich anwendbar. Sie genügt den Anforderungen einer lasttragenden Osteosynthese. Der Mandible External Fixator ist nicht zuletzt aufgrund der geringen Weichgewebstraumatisierung eine interessante Option bei multifragmentären Frakturen oder Defektzonen, die mit einer reduzierten Knochenqualität und/oder Weichgewebsdefiziten einhergehen.

\section{External Pin Fixation for the Temporary Bridging of Defect Zones in the Mandible}

External pin fixation of the mandible is a temporary alternative to conventional internal load-bearing osteosynthesis. The device consists basically of three titanium components: 1 ) pins or Schanz screws, 2) preshaped rods, 3) adjustable combination clamps with snap-on mechanisms. The whole framework can be assembled in the "visor-style" mode or alternatively in the "modular technique". The use of the external fixator is restricted to particular indications: a) pathological fractures in infected osteoradionecrosis, b) major resectional defects due to ablative tumour surgery, c) emergency care of mandible fractures in multipiece, heavy comminutions. Our experience shows that the technique is safe and reliable. External pin fixation in the mandible guarantees for loadbearing osteosynthesis. In conclusion, due to atraumatic soft-tissue management, the external mandibular fixator offers an interesting option in comminution or defect zones associated with reduced bone quality and/or soft tissue alterations and loss.

\section{Einleitung und historischer Überblick}

Der Gebrauch eines Fixateur externe stellt heute eine eher ungewöhnliche Differenzialtherapie zur Stabilisierung des Unterkiefers von außen dar und ist

OP-JOURNAL 2013; 29: 147-155

(c) Georg Thieme Verlag KG Stuttgart · New York DOI http://dx.doi.org/10.1055/s-0033-1350791 einigen speziellen Indikationen vorbehalten (Tab. 2). Die Idee einer perkutanen knochengetragenen Halterung kam zunächst im Bereich der Frakturbehandlung der Extremitäten auf und ist u.a. auf Wurzer (1853), Malgaigne (1853), von Langenbeck (1888) oder Parkhill (1897) zurückzuführen [6-26]. 1907 berichtete Lambotte als Erster von einer systematischen Anwendung. Während der Zeit des Zweiten Weltkriegs wurde der Fixateur externe dann regelmäßig zur Versorgung von Unterkieferfrakturen eingesetzt. Interessanterweise wurde dabei das Instrumentarium nicht nur aus der Extremitätenchirurgie, sondern auch aus der Veterinärmedizin transferiert. Das ursprüngliche Equipment wurde jedoch als sperrig, unhandlich und unhygienisch beurteilt und bald umfangreich modifiziert $[21,23]$ und die Methodik als „pin fixation“ tituliert [27]. Charakteristisch waren die unilaterale Anlage entlang der Außenfläche des Unterkiefers sowie die Modulartechnik. Mindestens 2 Pins wurden dabei je Fragment inseriert und mithilfe einer Klemme mit einem den Unterkiefer in der Transversalebene umfassenden Metallstab verbunden. Dadurch wurden stets jeweils 2 Fragmente miteinander verbunden, womit jeweils kleinere Untereinheiten entstanden. Diese Untereinheiten wurden schließlich über einen übergreifenden Verbindungsstab gerahmt und zusammengefügt. Modifikationen dieser Technik wurden von Converse und Waknitz (1942) und Morris (1949) beschreiben $[6,20]$. Nachdem der Gebrauch des Fixateur externe für den Unterkiefer in den 1940er-Jahren seinen Ursprung hatte, wurde die Technik bis Anfang der 1970er-Jahre immer populärer $[13,19,1-28]$. Indikationen waren dislozierte Frakturen, Frakturen des atrophen Unterkiefers, Frakturen im Bereich der nicht bezahnten Unterkieferabschnitte wie Kieferwinkel und aufsteigender Unterkieferast, Trümmerfrakturen, pathologische Frakturen und Unterkieferdefekte - insgesamt Situationen, in denen eine Versorgung mit transoral applizierten Kieferbruchschienen oder Prothesen nicht möglich war. Ab Mitte der 1970er-Jahre stagnierte dann die Verwendung des Fixateur externe. Erst Mitte der 1980er-Jahre erschienen wieder eine Reihe neuer Publikationen, in welchen über die Verwendung von Stäben aus Stahl, sogenannte „steel rods“, berichtet wird [26,5-32]. Unter diesen befand sich auch der sogenannte „clamp 


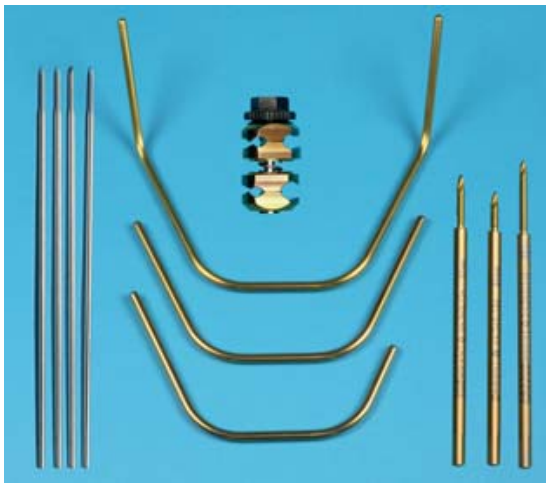

Abb. 1 Die Grundelemente des Mandible External Fixator: Schanz-Schrauben in 2 Durchmessern, vorgeformte Verbindungsstäbe, Klemmbacke.

fixator mandible type“, der von Spiessl [26] entwickelt wurde und auf einem bestehenden AO-Fixateur für den Extremitätenbereich basierte. Dieses System wies ein vergleichsweise einfaches Design auf und bestand im Wesentlichen aus 3 Komponenten: vorgeformten Metallstäben, Schanz-Schrauben und Klemmbacken als Verbindungselemente. Dies sind auch die in einem heute gebräuchlichen und später in diesem Beitrag beschriebenen External Mandible Fixator vorzufindenden Elemente [11].

Nachdem in den 1990er-Jahren die Verwendung interner rigider Osteosynthesen im Unterkieferbereich immer populärer wurde, verschwand die Anwendung des Fixateur externe nach und nach und wurde letztlich gar als Anachronismus betrachtet. Dies mündete darin, dass der externe Fixateur nach Spiessl [26] aus dem Portfolio des Herstellers genommen wurde. Erst Ende der 2000er-Jahre kam es zu einer Renaissance des Fixateur externe für den Unterkiefer, welcher von der Firma Synthes (Synthes Maxillofacial ${ }^{\circledR}$, Paoli, PA, USA) auf der Basis von Spiessl beschriebenen und weiter modifizierten Variante wieder aufgelegt wurde. Dieses System, bestehend aus innovativen Bestandteilen wie einem leichtgewichtigen Titankonstrukt, sollte sowohl ein komfortables Handling ermöglichen als auch ein stabiles Gerüst bilden, um eine mechanisch rigide externe Überbrückung von Defektzonen im Unterkiefer gewährleisten zu können.

\section{Methodik}

Die grundlegenden Elemente des im Folgenden beschriebenen Mandible External Fixator (Fa. Synthes Maxillofacial ${ }^{\circledR}$, Paoli, PA, USA) sind:

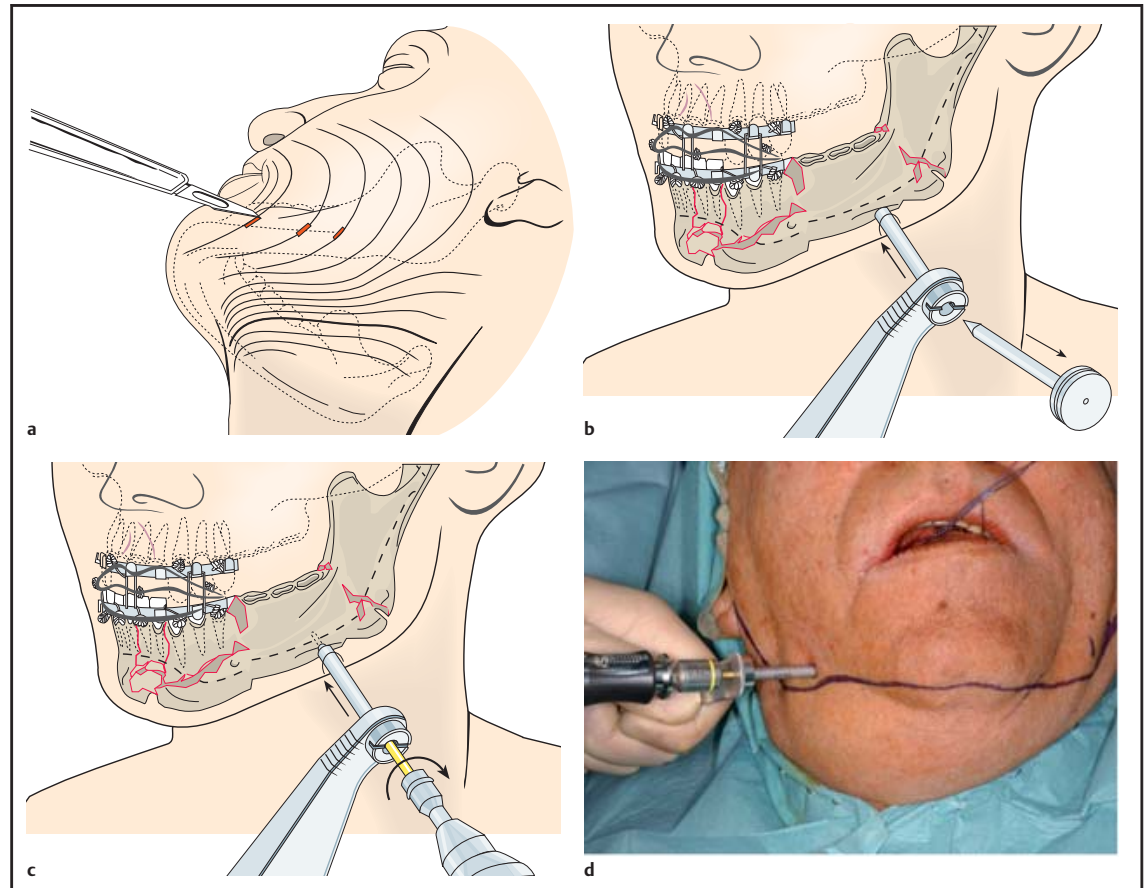

Abb. $\mathbf{2 a}$ bis d Einbringen der Pins bzw. Schanz-Schrauben: a Stichinzision parallel der Relaxed Skin Tension Lines (RSTL). b Nach stumpfer Präparation eines Weichgewebskanals und Einbringen eines Trokars mit Bohrhülse wird der Trokar wieder entfernt und über die verbleibende Bohrhülse der Pin bzw. die Schanz-Schraube im c „self-drilling“ Modus oder im d „self-tapping“ Modus eingedreht.

1. Pins oder Schanz-Schrauben aus Titan, „self-drilling“ oder „self-tapping“ für eine perkutane Anwendung (Durchmesser 2,5 mm/4,0 mm; Länge 50/60/ $70 / 80 \mathrm{~mm}$ )

2. Vorgeformte Verbindungsstäbe aus Titan, „connecting bars“ (Durchmesser $4,0 \mathrm{~mm}$ ) in unterschiedlicher Ausdehnung, um die Unterkieferanatomie nachzuahmen:

- Hälfte eines Unterkieferkorpus

- Drei Viertel eines Unterkieferkorpus

- kompletter Halbkreis eines Unterkieferkorpus

- kompletter Halbkreis eines Unterkieferkorpus inklusive aufsteigender Äste

3. Verstellbare, kombinierte Klemmen mit Schnappmechanismus aus Titan, um die Pins oder Schanz-Schrauben mit den vorgeformten Stäben zu verbinden.

Die Klemmbacken weisen 2 Paar nach lateral hin offene Klemmschlitze auf. In diesen können Pins von 2,5 mm oder 4,0 mm Durchmesser sowie Stäbe mit $4,0 \mathrm{~mm}$ Durchmesser über einen Schnappmechanismus eingebracht werden. Kontermuttern versteifen die Klemmschlitze gegeneinander, was $\mathrm{zu}$ einer rotationsstabilen Verbindung zwischen Stab und Pins führt (Abb. 1).
Vor der Verwendung des Fixateur externe können die wesentlichen Fragmente bzw. Segmente mittels rigider mandibulomaxillärer Fixation (MMF) immobilisiert werden.

Die Pins bzw. Schanz-Schrauben werden transbukkal inseriert. Dabei helfen Trokare und Bohrhülsen, die Weichgewebe nicht zu verletzen. Die Länge der Bohrhilfen resultiert aus der Dicke des Weichgewebemantels und der zuvor festgelegten Pinlänge. Dabei ist der Unterkieferunterrand die geeignete Region, um die Pins bzw. Schanz-Schrauben anzubringen. Diese sollten bikortikal und senkrecht zur Außenfläche der Mandibula verankert werden. Abhängig von Knochenqualität und Fragmentgröße können die Schanz-Schrauben per Hand nach Vorbohrung und Längenmessung („,self-tapping“) oder direkt („self-drilling“) eingebracht werden (Abb. 2).

Prinzipiell können die Komponenten des Fixateur-externe-Systems in der Modulartechnik oder in „Visor-style-Technik zusammengefügt werden.

\section{„Visor-style“-Technik}

Die ersten beiden Schanz-Schrauben werden in beschriebener Weise transbukkal in die jeweils gegenüberliegen- 


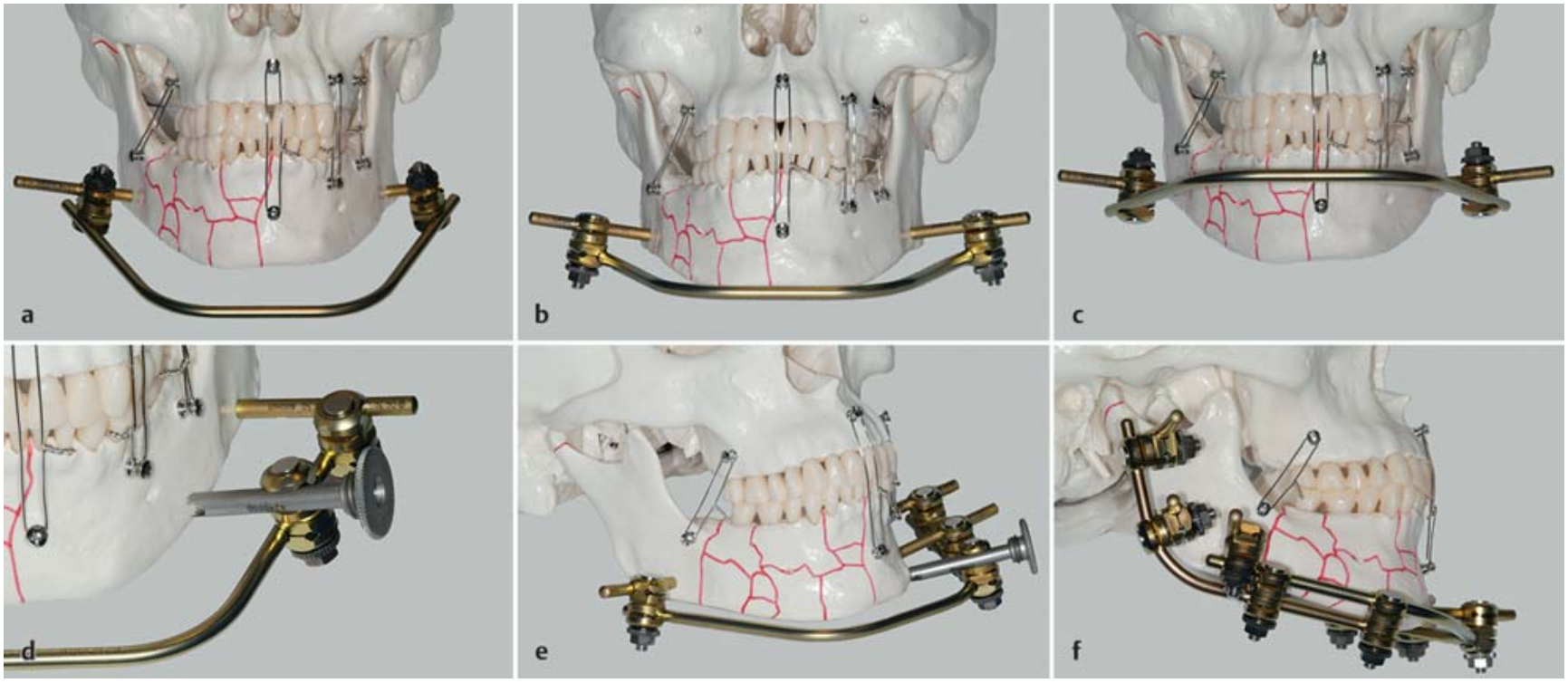

Abb. 3 „Visor-style“-Technik Reihe oben: feste mandibulomaxilläre Fixation über Drahtligaturen nach Reposition der Fragmente. Nach Insertion von 2 posterioren Pins wird der Mandibularbogen eingesetzt. Dieser ist nach oben und unten klappbar (a-c). Reihe unten: Durch eine 3. Klemmbacke wird ein Trokar eingeführt und die Position der 3. Schanz-Schraube festgelegt. Sind die Klemmbacken fest fixiert, ist eine visierartige Bewegung des Bogens nicht mehr möglich. Weitere Fragmente werden auf diese Art am Bogen fixiert. Die Rahmenkonstruktion kann als Monoframe (links und Mitte) oder Double-stacked Frame (rechts) ausgebaut werden.

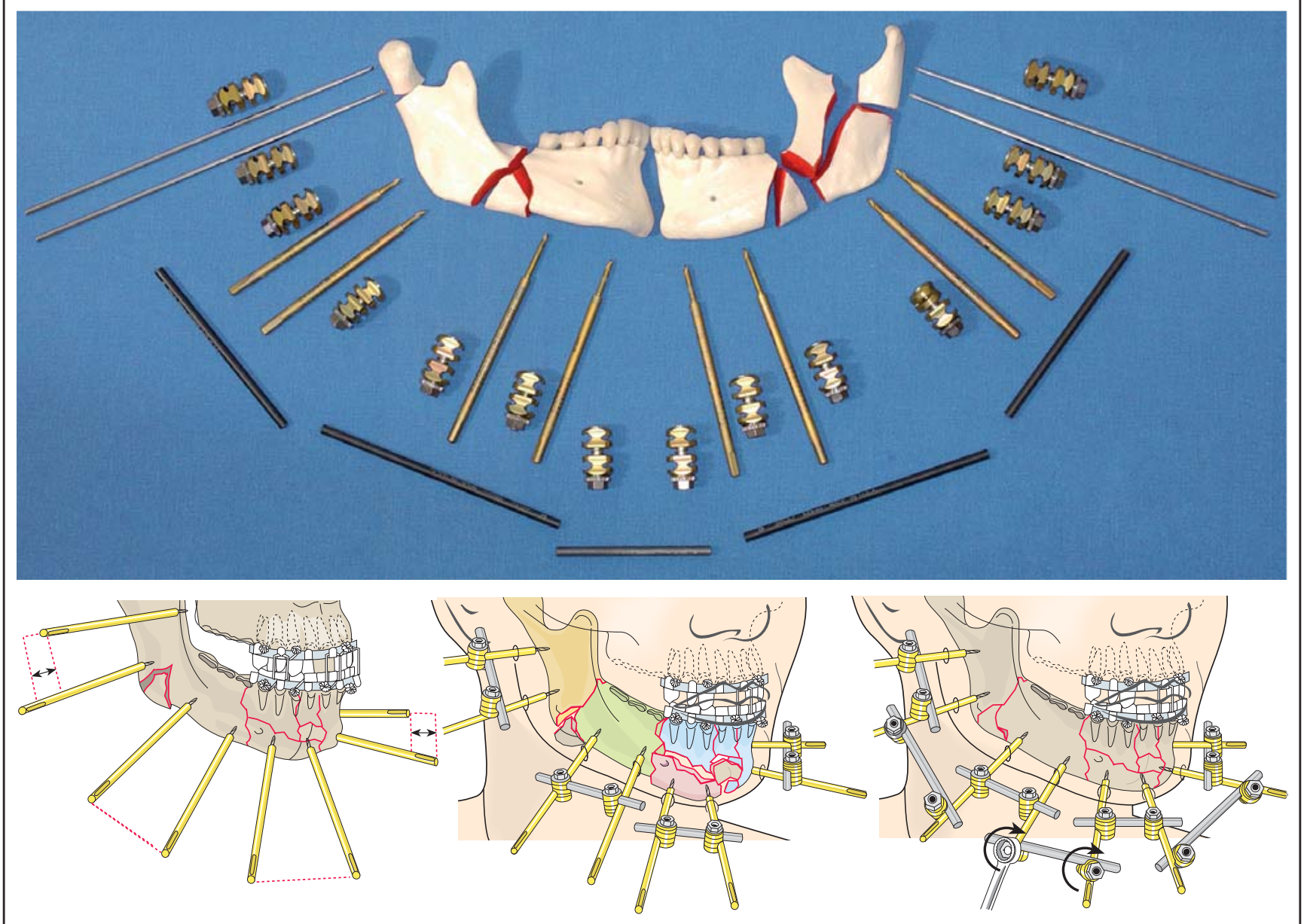

Abb. 4 „Modulartechnik“ - pro Fragment werden 2 Pins (2,5 mm oder 4 mm Durchmesser) inseriert, die über Karbonstäbe zuerst untereinander verbunden werden. Nach Reposition werden Untereinheiten geschaffen, die dann schrittweise zu einem festen Gesamtkonstrukt zusammengefügt werden. 


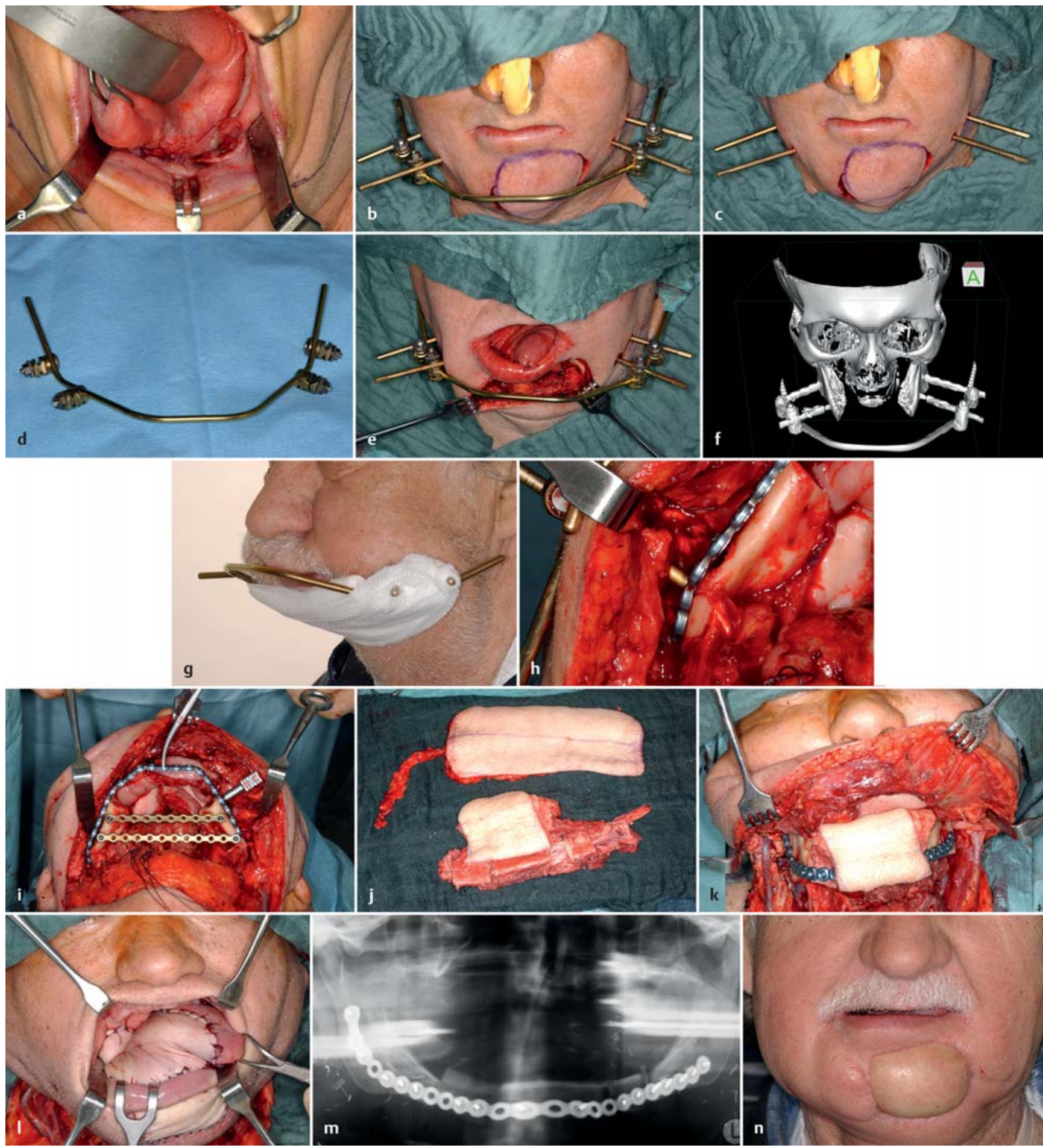

Abb. $\mathbf{5}$ a bis $\mathbf{n}$ Fallbeispiel 1: a ausgedehntes Mundbodenkarzinom mit Infiltration in den Unterkiefer und in die Zunge. b Nach Anlage eines Fixateur externe mit Pins im Bereich des dorsalen Unterkieferkorpus ist durch den Mandibularbogen die Tumorresektion im Kinnbereich nur erschwert möglich. c Der Mandibularbogen wird zur Tumorresektion entfernt. d Die Klemmbacken werden auf dem Mandibularbogen belassen und die genaue Position wird markiert, um im Anschluss an die Tumorresektion den Mandibularbogen wieder exakt platzieren zu können. e Fest montierter Mandibularbogen nach Tumorresektion. Gut zu erkennen ist der Unterkieferstumpf links. Die Zungenoberseite ist an der Unterlippe fixiert. Der Weichgewebedefekt am Kinn wird tamponiert. f 3-D-Rekonstruktion der Computertomografie mit Fixateur externe mit ausgedehntem knöchernen Unterkieferdefekt. g Verband zur Sicherung der Tamponade im Kinnbereich. Die sperrige Konstruktion stört die Patienten erstaunlicherweise kaum. h Im Rahmen der Rekonstruktion soll zur internen Fixation eine individuell vorgebogene Unterkieferrekonstruktionsplatte eingebracht werden. Aufgrund der Position eines Pins ist die Anbringung der Rekonstruktionsplatte am Unterkieferrand bei liegendem Fixateur nicht möglich. i Vor Abnahme des Fixateur externe werden die Unterkieferstümpfe daher mit 2 Osteosyntheseplatten stabilisiert. Die Rekonstruktionsplatte kann danach problemlos am Unterkieferrand fixiert werden. j Die Rekonstruktion des knöchernen Unterkiefers erfolgt mit einem mikrovaskulär reanastomosierten osteomyokutanen Fibulatransplantat. Intraoral wird ein A.-radialis-Lappen eingebracht. k Das Fibulatransplantat wird mit Osteosyntheseschrauben an der Rekonstruktionsplatte fixiert. Die Hautinsel dient zur Deckung des Defekts der äußeren Haut im Kinnbereich. I Direkt postoperativer Situs: Mundboden mit A.-radialis-Transplantat, Kinnbereich mit Hautinsel der Fibula. m Postoperatives Orthopantomogramm. Das Fibulatransplantat ist im mittleren Abschnitt erkennbar. n Zustand 3 Monate postoperativ. 
Tab. 1 Wichtige biomechanische Aspekte.

- Verwendung von Pins und SchanzSchrauben mit großem Durchmesser (4,0 mm).

- Insertion der inneren Pins nahe (ca. $10 \mathrm{~mm}$ Entfernung) des Defektbereichs und Insertion der äußeren Pins möglichst weit davon entfernt.

- Der Titanstab sollte - wenn möglich nicht zu weit von der Hautoberfläche entfernt angebracht werden.

den intakten Fragmente inseriert. Dies sollte in einem Bereich geschehen, der möglichst weit vom Defektgeschehen entfernt ist. Die Pins müssen dabei auf einer Ebene mit dem ausgewählten und konturierten Mandibularbogen zu liegen kommen. Dessen Enden werden mittels der Klemmbacken mit den posterior liegenden Pins verbunden. Der Mandibularbogen, der an den zuerst gesetzten Pins im Dorsalbereich des Unterkiefers bzw. an den Kieferwinkeln fixiert ist, erinnert an das Visier eines Helmes. Sind die Klemmbacken noch nicht fest geschlossen, kann dieses "Visier" in der Scharnierachse auf- und zugeklappt werden. Als nächstes wird die 3. Klemme in etwa $10 \mathrm{~mm}$ Entfernung vom Defekt an den Titanrahmen angebracht. Dann wird ein Trokar in die Klemmbacke eingesteckt und die gewünschte Position des Pins festgelegt. Nach Stichinzision der Haut unter Beachtung der Hautspannungslinien und stumpfem Eingehen bis auf die Knochenoberfläche wird der Pin durch den Trokar in den Knochen eingeschraubt. Die Klemme wird gelöst, die Bohrhülse entfernt und abschließend der in situ befindliche Pin durch Anziehen der Kontermutter fixiert. Durch die Insertion weiterer Pins wird das Gesamtkonstrukt fertiggestellt.

Zur Überbrückung kürzerer Defektregionen (ca. 4-5 cm) können schon 2 perkutane Pins bzw. Schanz-Schrauben auf jeder Seite des Rahmens für ausreichende Stabilität sorgen.

Ausgedehntere Defekte benötigen die Verwendung zusätzlicher Pins. Ein Titanstab oder Mandibularbogen wird in alleiniger Anwendung auch als „Monoframe“ bezeichnet. Er kann durch weitere Stäbe ergänzt werden, um zusätzliche Stabilität zu erreichen (Abb. 3).

Tab. 2 Indikationen für den Gebrauch eines Fixateur externe des Unterkiefers.

- pathologische Unterkieferfrakturen bei infizierter Osteoradionekrose, Malignomen, Osteomyelitis oder ausgedehntesten zystischen Formationen

- schwerwiegende multifragmentäre bzw. Trümmerfrakturen sowie Defektsituationen bspw. nach Schussverletzungen mit ausgedehnten Weichteilverletzungen

- Überbrückung segmentaler Unterkieferdefekte bei unklaren Resektionsrändern (R0?) bis zur Nachresektion bzw. sekundären Rekonstruktion

Notfallsituationen bei offenen Frakturen im Rahmen eines Polytraumas

\section{Modulartechnik}

Vor allem beim Vorliegen mehrerer Fragmente, bspw. bei Trümmerfrakturen, sollte der sogenannten Modulartechnik der Vorzug gegeben werde. Zunächst werden Untereinheiten geschaffen. Zwei Pins werden pro Fragment inseriert und über Klemmbacken und einen Stab miteinander fixiert. Dann kann ein Verbindungsstab lose mit Stab-zu-StabKlemmbacken zwischen 2 Untereinheiten angebracht werden. Nun kann die Reposition der Fragmente erfolgen und der Verbindungsstab fest fixiert werden. Auf diese Art und Weise werden jeweils benachbarte Untereinheiten miteinander verbunden, bis das Gesamtkonstrukt fertiggestellt ist. Als Nachteil dieser Technik sei hier angeführt, dass eine groß Menge von Hardware-Elementen notwendig ist, bis ein stabiles Gesamtkonstrukt fertiggestellt ist (Abb. 4).

Unabhängig davon, ob die „Visor-style“Technik oder die Modulartechnik zur Anwendung kommt, sollten wo immer möglich die hinlänglich bekannten biomechanischen Prinzipien berücksichtigt werden, um eine möglichst hohe Festigkeit des Fixateur-Gesamtkonstrukts sicherzustellen (Tab. 1).

\section{Fallbericht 1 (Abb. 5)}

Ein 58-jähriger Patient mit ausgedehntem Mundbodenkarzinom stellte sich zur operativen Tumortherapie mit Blockresektion unter Einschluss der Haut über dem Kinn, eines anterolateralen Unterkiefersegments, des Mundbodens und Anteilen der Zungenunterseite vor. Zur Stabilisierung und Fixierung der Position der Unterkieferstümpfe wurde ein External Mandible Fixator im Visor-style vor der Tumorresektion angebracht und zur Tumorresektion wieder abgenommen. Die Position der Klemmbacken wurde mit einem wasserfesten Stift auf dem Mandibularbogen und den Pins markiert und der Bogen nach der Tumorresektion wieder positioniert.
Die anatomisch korrekte Lage der Unterkieferstümpfe bietet im Falle einer Nachresektion sehr gute Zugangsmöglichkeiten zum Resektionsrand und verhindert eine Schrumpfung und den Kollaps der Weichgewebe.

Zwanzig Tage nach der Tumorresektion erfolgte die Nachresektion im Bereich der knöchernen Absetzungsränder, die Neck Dissection beidseits und die Rekonstruktion des Unterkieferdefekts nach vorheriger Planung mit einem mikrovaskulär reanastomosierten osteofasziokutanen Fibulatransplantat von rechts. Der Mundboden wurde mit einem revaskularisierten Radialislappen rekonstruiert. Vor der Abnahme des Fixateur externe wurden die Unterkieferstümpfe mit einer internen Fixation über 2 2.0-Unilock-Large-Profile-Platten (Fa. Synthes) stabilisiert. Der Fixateur externe wurde entfernt und eine zuvor anhand von CTAufnahmen vorgebogene, geometrisch vereinfachte Matrix-2.4-Rekonstruktionsplatte (Fa. Synthes) zur internen Defektüberbrückung mit Schrauben fixiert. Damit wurden die Stellung der Unterkieferstümpfe und die Position der Kiefergelenke aufrechterhalten.

\section{Fallbericht 2 (Abb. 6)}

Unserer Klinik wurde ein 23-jähriger Patient zugewiesen, der sich bei kämpferischen Auseinandersetzungen im Krisengebiet im Norden Afrikas im Jahr 2011 durch eine Schussverletzung mehrfragmentäre Unterkieferfrakturen anterolateral rechts, Frakturen im Bereich des Oberkiefers rechts, diverse Zahnfrakturen und ausgedehnte Weichgewebsverletzungen im Bereich der Wange rechts zugezogen hatte.

Bei der klinischen Untersuchung zeigten sich eine instabile osteosynthetische Versorgung der Unterkieferfrakturen mit Miniplatten und Drahtligaturen, freiliegende Knochenoberflächen und Zahnwurzelreste im Ober- und Unterkiefer. 

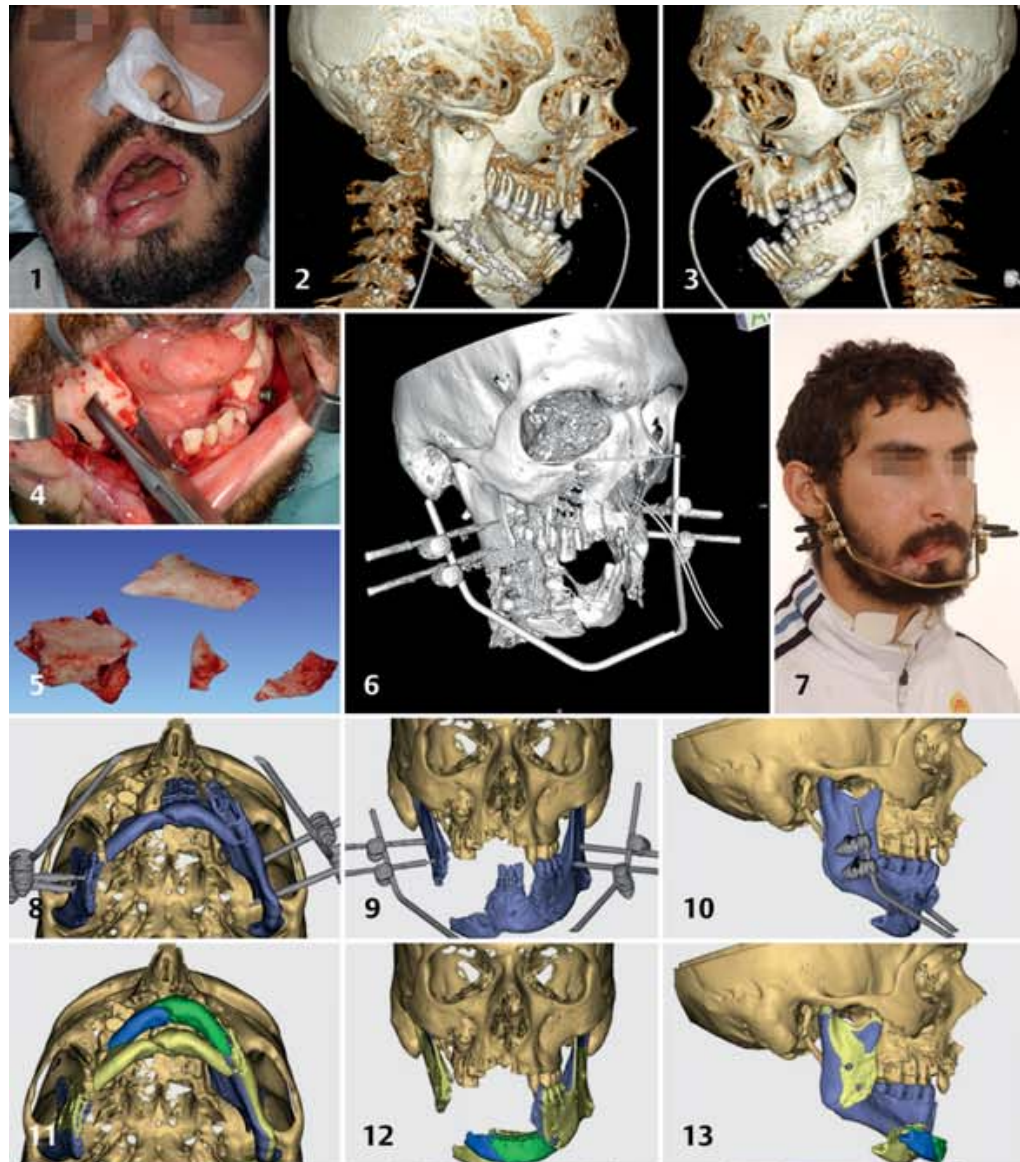

12
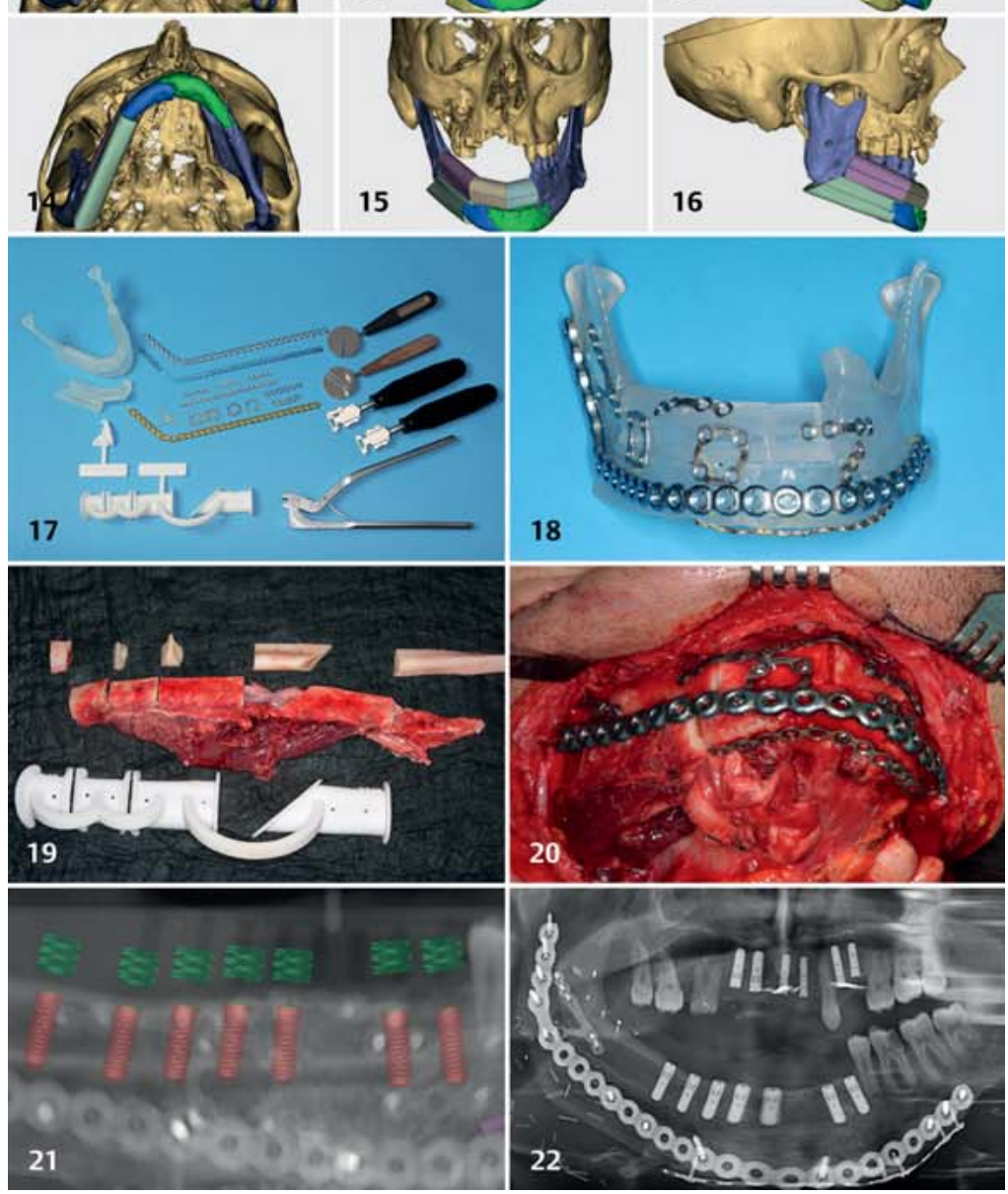
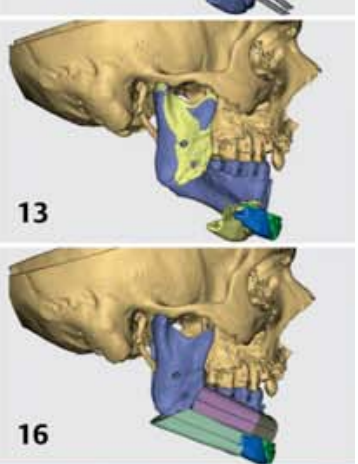

13

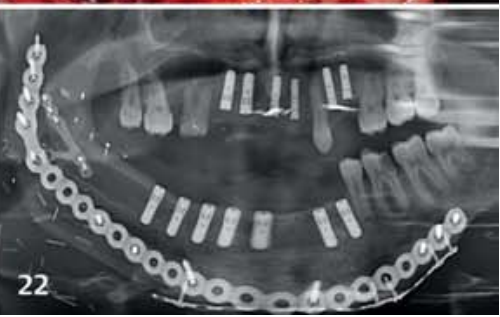

Abb. 6 Fallbeispiel 2: 20-jähriger Libyer nach Schussverletzung: 1-3 Klinisches Bild und 3-D-CT nach Erstversorgung in Tunis: fehlender Lippenschluss, in Fehlstellung osteosynthetisch versorgte Unterkiefermehrfragmentfraktur, unzureichende insuffiziente Osteosynthese mit zu klein dimensionierten Platten, feste mandibulomaxilläre Fixation links mit Draht. 4,5 Entfernung von Sequestern. Das gesamte Osteosynthesematerial wird entfernt. 611 Stabilisierung der Unterkieferstümpfe durch Anlage eines Fixateur externe im Bereich der aufsteigenden Unterkieferstümpfe beidseits bis zur Abheilung der intraund extraoralen Wunden. Die beiden Kinnfragmente werden in dislozierter Position belassen. 12-14 virtuelle Reposition der Kinnfragmente (gelb $\rightarrow$ grün, blau). 1516 virtuelle Planung der Unterkieferrekonstruktion mittels mikrovaskulär reanastomosiertem Fibulatransplantat. Die Höhe des Unterkiefers mit Alveolarfortsatz lässt sich im Korpusbereich durch eine Doppelung des Transplantats realisieren. Im Symphysenbereich wird der Alveolarfortsatz durch die einfache Höhe der Fibula rekonstruiert. 17 Patientenindividuelle STL-Modelle und Cutting Templates. Rekonstruktions- und Osteosyntheseplatten sowie Biegezangen und Schneidwerkzeuge. 18 Die Osteosyntheseplatten werden am STL-Modell vorgebogen. 19 Das Fibulatransplantat mit Gefäßstiel (rechts). Anhand des Cutting Guides werden die Abschnitte des Transplantats gesägt. 20 Die reponierten Kinnfragmente sowie der kaudale Anteil des Fibulatransplantats werden mittels Rekonstruktionsplatte fixiert. Der Alveolarfortsatz wird aus 3 Anteilen der Fibula geformt und mit Miniplatten fixiert. 21 Virtuelle Planung der Implantatpositionen im Unterkiefer. Die Implantate werden bikortikal in die Fibula eingebracht. Rot: Implantat, grün: Bohrhülse. 22 Röntgenkontrolle nach partieller Metallentfernung und Implantatinsertionen im Ober- und Unterkiefer zur dentalen Rehabilitation. 
Nach CT-Bildgebung erfolgten in einem ersten operativen Schritt im November 2011 ein Débridement, Sequestrotomien, Entfernung des Osteosynthesematerials und der Zahnwurzelreste sowie die Applikation eines Mandible External Fixators, um die Wundinfektion im Defektbereich auszuheilen. Zwei weichgewebegestielte Fragmente im basalen Kinnbereich wurden dabei erhalten.

Der knöcherne Unterkieferdefekt wurde zunächst belassen. Die knöcherne Rekonstruktion des Unterkiefers wurde auf Basis einer erneuten CT-Untersuchung geplant. Mit dem Datensatz konnte die virtuelle Konfiguration eines myoossären, vaskularisierten Fibulatransplantats von links im Sinne eines komplexen, mehrfach segmentierten, Over and under Barrel Grafts simuliert werden. Präoperativ wurde ein Outcome-Hybridmodell zur Präformierung der Osteosyntheseplatten angefertigt. Zur Osteosynthese wurden 2.0- und 2.4Matrix-Mandible-Rekon- und Traumaplatten am Modell vorgebogen. Die Überbrückung des großen Defekts und Rekonstruktion eines Alveolarfortsatzes war dadurch in ausreichender Höhe möglich. Im Dezember 2011 wurden die Kinnfragmente reponiert und der Alveolarfortsatz im anterolateralen Bereich mithilfe von 3 Fibulasegmenten aufgebaut, sowie der basale Unterkieferkorpusbereich rechts mit einem weiteren Fibulasegment wiederhergestellt.

Im Ergebnis hatten die skelettalen Verhältnisse im Defektbereich die originäre Dimension. Mithilfe einer 3-D-Implantatplanung erfolgte die kaufunktionelle Rehabilitation. Nach Erstellung eines virtuellen Set-ups mit der Software 3DIP (Gäßler Zahntechnik, Neu-Ulm) und nach Herstellung einer CT-Bohrschablone führten wir die geführte Implantation im Ober- und Unterkiefer durch. Das Osteosynthesematerial wurde im gleichen Eingriff teilweise entfernt.

\section{Eigene Erfahrungen}

Im Folgenden soll kurz eigene Erfahrungen [25], die bei der Anwendung des Fixateur externe gesammelt wurden, eingegangen werden. Bei folgenden Patientengruppen wurde ein External Mandible Fixator appliziert:

1. Palliation bei onkologischen Patienten

2. onkologische Patienten mit kurativem

Setting bei ausgedehnter Tumorgröße

3. Trümmerfrakturen des Unterkiefers
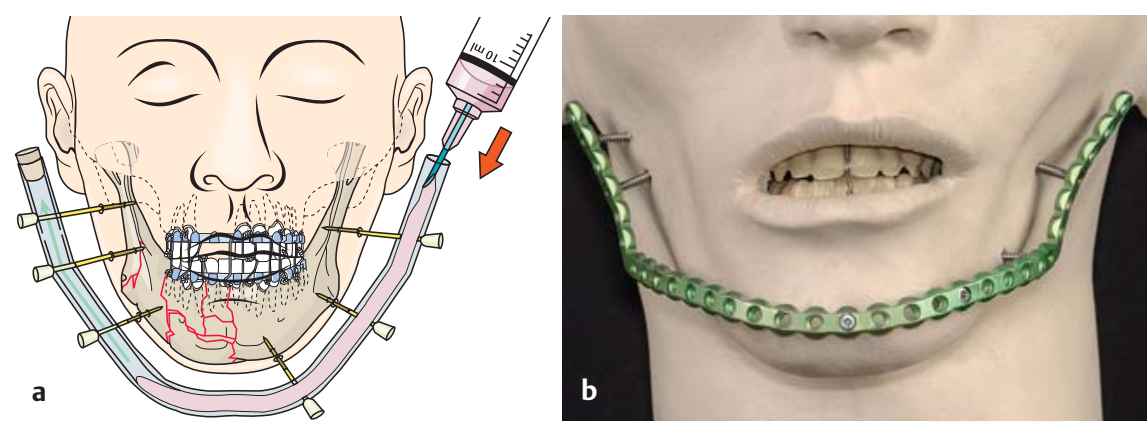

Abb. 7a und $\mathbf{b}$ a Ein endotrachealer Tubus mit Perforationen wird über die Pins gezogen und mit Autopolymerisat gefüllt. Während der Polymerisation müssen die Fragmente in der richtigen Position gehalten werden. b Alternativ zum Mandibularbogen wird eine Locking-Platte als Rahmen verwendet. Statt Pins werden überlange Locking-Schrauben verwendet.

Im gesamten MKG-LMU-Patientenkollektiv $(n=24)$ betrug die Zeit für die komplette Applikation des Fixateur externe zwischen 20 und 35 Minuten. Die Pininsertion erfolgte stets bikortikal und ließ sich atraumatisch durchführen. Die Pins waren dabei primärstabil.

Die Tragezeit des Fixateur betrug durchschnittlich 75 Tage (maximal 175 Tage). In diesen Zeiträumen trat keine Pinlockerung auf. Im Bereich der Hautdurchtrittsstellen kam es bei keinem Patienten zu einer Infektion. Speichelfisteln nach transparotidealer Pininsertion wurden nicht beobachtet. Ebenfalls nicht aufgetreten sind mechanische Probleme, die auf die Klemmen oder Stäbe zurückzuführen gewesen wären und zu Verschiebung der Untereinheiten geführt hätten.

Erstaunlicherweise fühlten sich die Patienten nicht sehr von der Apparatur gestört. Schlafgewohnheiten wurden entsprechend angepasst. Das Rasieren und die Pflege der Gesichtshaut erfolgten durch Familienangehörige oder den Pflegedienst.

Nach der Entfernung des jeweiligen Fixateurs resultierten kleinere narbige Einziehungen im Bereich der äußeren Haut, die von den Patienten aber als nicht störend empfunden wurden und mit der Zeit zunehmend unauffällig wurden.

\section{Diskussion}

Ein vollständig montierter und in Endposition fixierter Fixateur externe eignet sich prinzipiell zu einer winkelstabilen Osteosynthese des Unterkiefers und kann als Vorreiter der modernen winkelstabilen Plattensysteme betrachtet werden.
Die Biomechanik externer Fixationssysteme für den menschlichen Unterkiefer war indessen bis dato kaum Gegenstand wissenschaftlicher Betrachtungen [26, 24]. Zu dieser Thematik liegen aber Berichte aus Anwendungen beim Hund vor, welche jedoch nur teilweise auf den Menschen übertragbar sein dürften [7].

Unserer Meinung nach kann der Fixateur externe des Unterkiefers aufgrund seiner Materialeigenschaften und Dimensionen als lasttragende Osteosynthese angesehen werden [8].

Selbstverständlich sind die Indikationen, insbesondere vor dem Hintergrund moderner Plattensysteme, einigen Sondersituationen vorbehalten, bei denen Defekte oder defektähnliche Bereiche bzw. Frakturen vorliegen, ggf. in Kombination mit verminderter Knochenqualität und kompromittierten Weichgeweben [32,4, 29]. Dabei kann im Wesentlichen ein onkologisches Setting von einem traumatologischen Anwendungsbereich differenziert werden. Zu Erstgenanntem zählen pathologische Frakturen auf dem Boden infizierter Osteoradionekrosen nach vorangegangener Strahlentherapie im MKG- und HNO-Bereich oder ausgedehnte Tumorinfiltration im MKG-Bereich. Im traumatologischen Sektor können als Indikationen gelten: multifragmentäre bzw. Trümmerfrakturen in Kombination mit extensiver Weichgewebezerreißung [30] oder Weichgewebedefekten, problematischer Durchblutungssituation oder nicht absehbaren Weichgewebsnekrosen im initialen Management von Schussverletzungen, Explosionstraumata, Verbrennungen 3. Grades oder Stromunfällen. Weitere ungewöhnliche Indikationen können Defektsituationen auf dem Boden von Osteomyelitiden, ausgedehnten bisphos- 
phonatassoziierten Nekrosen oder extensiver Zysten sein.

Die externe Stabilisierung des Unterkiefers bei polytraumatisierten Patienten gewährleistet eine zügige notfallmäßige Versorgung zur temporären Stabilisierung, insbesondere bei gleichzeitig vorliegenden vital bedrohlichen Verletzungen. Zur definitiven Frakturversorgung mit exakter Reposition der Fragmente erfolgt dann im Intervall die Konvertierung auf interne Überbrückungsplatten.

Die Insertion von Schanz-Schrauben ist insgesamt als eine relativ einfache, komplikationsarme Prozedur anzusehen, wozu nur in sehr begrenzten Umfang eine stumpfe Weichgewebepräparation erfolgen muss. Größere chirurgische $\mathrm{Zu}-$ gänge in der Submandibular-, der Submental- oder Retromandibularregion können ebenso vermieden werden wie ausgedehnte subperiostale Präparation.

Bei allen unseren Tumorpatienten, die zuvor eine Radiotherapie erhalten hatten, ergaben sich weder Wundheilungsstörungen noch Entzündungsreaktionen nach Anlage des Fixateurs. Bei einem Teil bildeten sich vorbestehende intra- und/ oder extraorale Fisteln spontan zurück und es kam zu einer Weichgewebskonsolidierung. Dies erlaubte den späteren Austausch des Fixateur externe gegen eine entsprechende Unterkieferrekonstruktionsplatte.

Bei der Vorbereitung zur Pininsertion ist es wesentlich, auf eine Reposition der Hauptfragmente zu achten und diese entweder manuell oder mittels MMF zu sichern. Wird der Fixateur externe im „Visor-style“ (siehe oben) zusammengefügt, muss zunächst mit der Reposition der Fragmente begonnen werden. Dies ist wichtig, denn sobald der Mandibularbogen im Bereich des R. mandibulae einmal angebracht ist, können zusätzliche Pins nur noch entlang des als Monofixator fungierenden Konstrukts inseriert werden, was eine Fragmentreposition sehr limitiert.

In den Fällen, in denen ein definierter Defektbereich oder eine einzelne zertrümmerte Zone überbrückt werden soll, sind die Hauptfragmente in der Regel einfach zu reponieren. Diese Fälle eignen sich besonders für die „Visorstyle“-Technik.

Anders verhält es sich beim Vorliegen multipler, dislozierter Fragmente. Hier ist der Modulartechnik Vorzug zu geben, wobei die einzelnen Untereinheiten Stück für Stück miteinander zu einem Gesamtkonstrukt verbunden werden müssen. Hierbei bestehen deutlich mehr Freiheitsgrade bei der Pinplatzierung. Nachteilig wirken sich naturgemäß die Größe und das hohe Gewicht der Gesamtkonstruktion aus.

Auf zwei bisher hier nicht erwähnte Fixateur-externe-Verfahren soll noch kurz eingegangen werden (Abb.7). Bei der 1. Variante werden die einzelnen Pins mit einem Autopolymer verblockt [29]. Dabei wird bspw. ein endotrachealer Tubus oder ein großlumiger Absaugschlauch mit entsprechenden Perforationen versehen über die Pins gezogen und mit einem flüssigen Autopolymerisat gefüllt. Für ausgedehntere und komplexere Defekt- und Trümmerzonen scheint diese Alternative jedoch eher nicht geeignet, da hierfür mehrere Assistenten benötigt werden, welche die Fragmente während der Polymerisation in Position halten.

Behelfsweise kann auch eine Rekonstruktionsplatte als Fixateur externe Verwendung finden. Die Platte wird in kurzem Abstand über der Haut des Unterkiefers ankonturiert, und die reponierten Fragmente werden mithilfe von langen perkutan geführten LockingSchrauben an dieser Platte fixiert [31]. Nach der Stichinzision und Unterminieren der Haut ist zur Schraubeninsertion ein Vorbohren des Knochens zu empfehlen.

Fixateur-externe-Anwendungen können für Monate im Unterkiefer in situ verbleiben $[13,26,2-18]$.

Lockerungen von Pins oder mechanische Probleme im Rahmensystem kommen kaum vor. Das Fixateur-Prinzip als externe, lasttragende Osteosynthese kann auch additiv zur internen Fixation durch Miniplatten eingesetzt werden, bis die knöcherne Konsolidierung und Stabilisierung einsetzt. Langzeitanwendungen kommen besonders bei kurativen oder palliativen onkologischen Fällen infrage. So können bspw. bei sehr ausgedehnten, aber noch operablen Tumoren vor rekonstruktiven Maßnahmen die definitiven histopathologischen Ergebnisse abgewartet werden (s. Falldarstellung 1). Nachresektionen können jederzeit erfolgen. Falls erforderlich, können Pins dazu neu positioniert werden.
Steht im Verlauf die Rekonstruktion mit mikrovaskulären Knochentransplantaten an, ist eine bildgebende Evaluation der aktuellen Situation mittels Computertomografie ohne störende Artefaktbildung in der Regel möglich.

Somit steht auch einer computerassistierten Planung, ggf. in Verbindung mit computergestützt hergestellten Rekonstruktionsplatten oder Osteotomieschablonen, nichts im Weg.

Nicht zuletzt bietet sich die Verwendung des External Mandible Fixators im Militärbereich an. So ist es möglich, in Kampfeinsätzen oder auch nach terroristischen Anschlägen in einem improvisierten medizinischen Umfeld unter Zeitdruck eine schnell durchführbare und suffiziente, lasttragende Stabilisierung bei Trümmerfrakturen im Unterkiefer zu erreichen. Diesbez. sei erwähnt, dass die US-Armee den externen Unterkiefer-Fixateur als „combat medical device“ klassifiziert.

\section{Fazit}

Die oben genannten Beispiele zeigen, dass die Verwendung eines Fixateur externe im Unterkieferbereich in ausgewählten Fällen eine wertvolle Therapieoption darstellen kann. Dies trifft in erster Linie für die Überbrückung von defektähnlichen Zonen bei Trümmerfrakturen oder Defekten zu, die mit einer reduzierten Knochenqualität und/oder stark verletzten Weichgeweben einhergehen. Ursachen solcher Defekte sind in unserem klinischen Alltag typischerweise pathologische Frakturen im Zusammenhang mit infizierten Osteoradionekrosen nach Strahlentherapie im KopfHals-Bereich sowie Schussverletzungen. Es kann eine schnelle, mechanisch lasttragende und zuverlässige Unterkieferstabilisierung erreicht werden, ohne dass ausgedehntere chirurgische Zugänge mit umfangreicher Weichgewebedissektion zum Einbringen von Rekonstruktionsplatten benötigt werden.

Bei Polytraumapatienten stellt die Stabilisierung des Unterkiefers mittels Fixateur externe eine schnell durchführbare und zuverlässige Notfalloption dar. Dabei sollte der Modulartechnik bei ausgedehnten multifragmentären Trümmerfrakturen der Vorzug gegeben werden. 


\section{Literatur}

1 Becker E. [A set of instruments for extracutaneous osteosynthesis of mandibular fractures with the use of plastic products]. Chirurg 1958; 29: 63-67

2 Bergman SA, Elias EG, Didolkar MS et al. Maintenance of function and esthetics after partial mandibulectomy without bone grafting. J Oral Surg 1981; 39: 421-425

${ }^{3}$ Birke WP. Methoden zur Verbesserung der Fragmentadaptation bei der perkutanen Osteosynthese mit Kunststoffbrücken. Dtsch Stomatol 1973; 23: 721-725

${ }^{4}$ Braidy HF, Ziccardi VB. External fixation for mandible fractures. Atlas Oral Maxillofac Surg Clin North Am 2009; 17: 45-53

${ }^{5}$ Caldani P, Eleuteri M, Feliciano $S$ et al. [Treatment of fractures of the mandible: a new type of external fixator]. Minerva Chir 1989; 44: 1681-1683

${ }^{6}$ Converse JM, Waknitz FW. External skeletal fixation in fractures of the mandibular angle. J Bone Joint Surg 1942; 24: 154-160

7 Cook WT, Smith MM, Markel MD et al. Influence of an interdental full pin on stability of an acrylic external fixator for rostral mandibular fractures in dogs. Am J Vet Res 2001; 62: 576-580

${ }^{8}$ Cornelius CP, Augustin JB, Sailer LK. External pin fixation for stabilization of the mandible-comeback of a method: historical review and first experiences with the 'mandible external fixator'. Oral Maxillofac Surg 2009; 13: $1-14$

${ }^{9}$ Fleming ID, Morris JH. Use of acrylic external splint after mandibular resection. Am J Surg 1969; 118: 708-711

10 Gabka J. Indikationen und Ergebnisse des extraoralen Schraubenschienenverbandes nach Becker. Fortschr Kiefer Gesichtschir 1975; 19: 117-119

11 Gibbons AJ, Mackenzie N, Breederveld RS. Use of a custom designed external fixator system to treat ballistic injuries to the mandible. Int J Oral Maxillofac Surg 2011; 40: 103-105

12 Gilles $H$, Millard D. Surgery of the mandible. Boston: Little, Brown and Company; 1957

13 Ginestet G. Le "fixateur externe» dans le traitement des fractures du maxillaire infe- rieur. Rev Odontol Stomatol Maxillofac 1946; 10: 455-460

14 Hierholzer G, Allgöwer M, Rüdi T. Fixateur-externe-Osteosynthese. Berlin: Springer; 1985

15 Janecka IP. External stabilization of the mandible with the Mini-H-Fixator. Plast Reconstr Surg 1984; 73: 840-842

${ }^{16}$ Lambotte A. L'intervention operatoire dans les fractures. In: Lambotte A, Hrsg. L'intervention opératoire dans les fractures. Bruxelles: Lamertin H; 1907

17 Losapio PL, Amaddeo P, Faldi F. [The use of minifixers-compressors in the treatment of fractures of the mandible]. Minerva Stomatol 1988; 37: 287-290

18 MacIntosh RB. Morrischer Apparat bei der Behandlung des Kieferbruches. Fortschr Kiefer Gesichtschir 1975; 19: 114-116

${ }^{19}$ Mathis $H$. Die Behandlung der Frakturen der zahnlosen Kiefer. Fortschr Kiefer Gesichtschir 1956; 2: 92-97

${ }_{20}$ Morris JH. Biphase connector, external skeletal splint for reduction and fixation of mandibular fractures. Oral Surg Oral Med Oral Pathol 1949; 2: 1382-1398; illust

21 Mowlem R, Macgregor A, Buxton JLD et al. External pin fixation for fractures of the mandible. The Lancet 1941; 238: 391-393

22 Pasturel A, Bellavoir A, Cantaloube D et al. [External fixation devices in maxillofacial surgery]. Rev Stomatol Chir Maxillofac 1984; 85: 426-428

23 Pohl L. Extra-oral splinting of the edentulous mandible. The Lancet 1941; 238: 389-391

24 Schüle $H$. Schienung von Unterkiefrakturen mittels extraoraler Verschraubung. Dtsch Zahnärztl Zeitschr 1957; 12: 933-944

25 Smolka W, Mast G, Ehrenfeld $M$ et al. The use of external pin fixation for stabilization of the mandible in recurrent squamous cell carcinoma of the oral cavity invading the mandible. Oral Oncology 2013 (submitted)

26 Spiessl B. Äussere Schienung: Fixateur externe. In: Spiessl B, Hrsg. Osteosynthese des Unterkiefers: Manual der AO-Prinzipien. Kap. 4.2.2, Berlin: Springer; 1988: 67-92

27 Thoma KH. Methods of fixation of jaw fractures and their indications. J Oral Surg (Chic) 1948; 6: 125-134
28 Ullik $R$. [Indication for nailing of jaw fractures according to Roger Anderson]. Zentralbl Chir 1953; 78: 1012-1014

29 Wessberg GA, Schendel SA, Epker BN. Monophase extraskeletal fixation. J Oral Surg 1979; 37: 892-896

30 Wilkening MW, Patel PA, Gordon CB. External fixation in a low-velocity gunshot wound to the mandible. J Craniofac Surg 2012; 23: e418-e419

31 Woon CY, Wong MK, Howe TS. LCP external fixation-external application of an internal fixator: two cases and a review of the literature. J Orthop Surg Res 2010; 5: 19

32 Zorman D, Godart PA, Kovacs B et al. Treatment of mandibular fractures by external fixation. Oral Surg Oral Med Oral Pathol 1990; 69: 15-19

\section{Dr. med. dent.}

Florian Andreas Probst

Assistenzarzt

Dr. med. Dr. med. dent.

Wenko Smolka

Oberarzt

Frank Tolksdorf

Assistenzarzt

Dr. med. Dr. med. dent.

Gerson Mast

Leitender Oberarzt

Prof. Dr. med. Dr. med. dent.

Carl-Peter Cornelius

Oberarzt

Klinik und Poliklinik für Mund-,

Kiefer- und Gesichtschirurgie

Klinikum der Universität München

Ludwig-Maximilians-Universität

München

Lindwurmstraße 2 a

80337 München

florian.probst@med.uni-muenchen.de 